OPEN ACCESS

Edited by: Kelly Anne Allott,

University of Melbourne, Australia

Reviewed by:

Minah Kim,

Seoul National University Hospital,

South Korea

Yuko Higuchi,

University of Toyama, Japan

*Correspondence:

Kiyoto Kasai

kasaik-tky@umin.net

Specialty section: This article was submitted to

Schizophrenia,

a section of the journa

Frontiers in Psychiatry

Received: 18 March 2020

Accepted: 21 July 2020

Published: 03 August 2020

Citation:

Fujioka M, Kirihara K, Koshiyama D, Tada M, Nagai T, Usui K, Morita S, Kawakami S, Morita K, Satomura Y, Koike S, Suga M, Araki T and Kasai K (2020) Mismatch Negativity Predicts

Remission and Neurocognitive Function in Individuals at Ultra-High Risk for Psychosis.

Front. Psychiatry 11:770. doi: 10.3389/fpsyt.2020.00770

\section{Mismatch Negativity Predicts Remission and Neurocognitive Function in Individuals at Ultra-High Risk for Psychosis}

\author{
Mao Fujioka ${ }^{1}$, Kenji Kirihara ${ }^{1}$, Daisuke Koshiyama ${ }^{1,2}$, Mariko Tada $^{1,3}$, Tatsuya Nagai ${ }^{1,4}$, \\ Kaori Usui ${ }^{1}$, Susumu Morita ${ }^{1}$, Shintaro Kawakami ${ }^{1}$, Kentaro Morita ${ }^{5}$, \\ Yoshihiro Satomura ${ }^{1}$, Shinsuke Koike ${ }^{1,3,6,7,8}$, Motomu Suga ${ }^{1,9}$, Tsuyoshi Araki ${ }^{1}$ \\ and Kiyoto Kasai ${ }^{1,3,6,8^{*}}$
}

1 Department of Neuropsychiatry, Graduate School of Medicine, The University of Tokyo, Tokyo, Japan, 2 Department of Psychiatry, University of California San Diego, La Jolla, CA, United States, ${ }^{3}$ The International Research Center for Neurointelligence (WPI-IRCN), University of Tokyo Institutes for Advanced Study (UTIAS), The University of Tokyo, Tokyo, Japan, ${ }^{4}$ Department of Psychiatry, Kawamuro Memorial Hospital, Joetsu, Japan, ${ }^{5}$ Department of Rehabilitation, Graduate School of Medicine, The University of Tokyo, Tokyo, Japan, 6 University of Tokyo Institute for Diversity and Adaptation of Human Mind (UTIDAHM), The University of Tokyo, Tokyo, Japan, ${ }^{7}$ Center for Evolutionary Cognitive Sciences, Graduate School of Art and Sciences, The University of Tokyo, Tokyo, Japan, ${ }^{8}$ UTokyo Center for Integrative Science of Human Behaviour (CiSHuB), The University of Tokyo, Tokyo, Japan, ${ }^{9}$ Graduate School of Clinical Psychology, Teikyo Heisei University, Tokyo, Japan

Background: In the early intervention in psychosis, ultra-high risk (UHR) criteria have been used to identify individuals who are prone to develop psychosis. Although the transition rate to psychosis in individuals at UHR is $10 \%$ to $30 \%$ within several years, some individuals at UHR present with poor prognoses even without transition occurring. Therefore, it is important to identify biomarkers for predicting the prognosis of individuals at UHR, regardless of transition. We investigated whether mismatch negativity $(\mathrm{MMN})$ in response to both duration deviant stimuli $(\mathrm{dMMN})$ and frequency deviant stimuli (fMMN) could predict prognosis, including remission and neurocognitive function in individuals at UHR.

Materials and Methods: Individuals at UHR $(n=24)$ and healthy controls $(H C ; n=18)$ participated in this study. In an auditory oddball paradigm, both dMMN and fMMN were measured at baseline. Remission and neurocognitive function after $>180$ days were examined in the UHR group. Remission from UHR was defined as functional and symptomatic improvement using the Global Assessment of Functioning (GAF) score and Scale of Prodromal Symptoms (SOPS) positive subscales. Neurocognitive function was measured using the Brief Assessment of Cognition in Schizophrenia (BACS). We examined differences in MMN amplitude at baseline between those who achieved remission (remitters) and those who did not (non-remitters). Multiple regression analyses were performed to identify predictors for functioning, positive symptoms, and neurocognitive function.

Results: Compared with the $\mathrm{HC}$ group, the UHR group had a significantly attenuated dMMN amplitude ( $p=0.003$ ). In the UHR group, GAF scores significantly improved during the follow- 
up period (mean value 47.1 to $55.5, p=0.004$ ). The dMMN amplitude at baseline was significantly larger in the remitter $(n=6)$ than in the non-remitter group $(n=18)(p=0.039)$. The total SOPS positive subscale scores and fMMN amplitude at baseline could predict BACS attention subscore at the follow-up point (SOPS positive subscales, $p=0.030$; $f M M N, p=$ 0.041).

Conclusion: Our findings indicate that $\mathrm{dMMN}$ and $\mathrm{fMMN}$ predicted remission and neurocognitive function, respectively, in individuals at UHR, which suggests that there are both promising biomarker candidates for predicting prognosis in individuals at UHR.

Keywords: mismatch negativity, ultra-high risk for psychosis, longitudinal study, remission, neurocognitive function

\section{INTRODUCTION}

Early intervention for individuals with schizophrenia is important, as the duration of untreated psychosis is known to predict the outcomes of schizophrenia (1). Furthermore, detection of schizophrenia before the onset of psychosis may lead to its prevention. However, identifying individuals who will develop psychosis at a later time is difficult at the prodromal stage because the symptoms are not disease-specific. Consequently, the ultra-high risk for psychosis (UHR) criteria, which include the brief intermittent psychotic syndrome (BIPS), attenuated positive symptom syndrome (APS), and genetic risk and deterioration syndrome (GRDS) assessments were developed $(2,3)$ and have been used (4) to identify individuals at high risk for psychosis.

Previous studies that have investigated the prognosis of individuals at UHR have mostly focused on the transition to psychosis. When the concept of UHR criteria was introduced, the transition rate in the first year was $40 \%$ to $50 \%(2,3)$. However, a recent meta-analysis showed a decreasing trend of $22 \%$ and $36 \%$ at the one-year and three-year follow-up time-points, respectively (4). Although many individuals at UHR do not transition to psychosis (non-converters), their prognosis is not necessarily good. A meta-analysis by Simon et al. reported that $73 \%$ of individuals at UHR did not transition to psychosis within 2 years of follow-up, and the percentage of non-converters who achieved remission from UHR status was 46\% (5). In a six-year follow-up study of individuals who met the UHR or basic symptoms (BS) criteria, which is another tool for risk assessment for psychosis, approximately $40 \%$ of the participants achieved full remission from UHR symptoms (6). Further, the functional prognosis is also poor. Another six-year, longitudinal, structural magnetic resonance imaging study of UHR or BS individuals reported that more than half of the participants had poor functional outcomes with a modified Global Assessment of Functioning scale score of $<65$ (7). A longitudinal study examining the natural history of 111 non-converted medication-naive individuals at UHR reported an improvement of baseline social and role functions over the course of 2 years. However, these functions were still significantly lower compared to those of nonpsychiatric participants (8). In summary, the prognosis of individuals at UHR is symptomatically and functionally poor, even if they do not transition to psychosis. Therefore, it is important to investigate and identify biomarkers for predicting prognoses and allowing for early intervention for individuals at UHR, regardless of whether they transition to psychosis.

To date, various indices have been studied to predict the transition to psychosis in individuals at UHR. Mismatch negativity (MMN), a negative component of event-related potentials (ERP) elicited by infrequent deviant stimuli occurring within a series of frequent standard stimuli, is considered a promising biomarker among other ERP components (9). In schizophrenia, the MMN amplitude decreases with a large effect size $(10,11)$. Moreover, MMN deficiency, which reflects functional impairment of N-methyl-D-aspartate receptors $(12,13)$, is associated with neurocognitive $(14,15)$ and functional decline $(16,17)$.

Although MMN is a promising biomarker for predicting the transition to psychosis $(18,19)$, it remains unclear whether MMN predicts the prognosis of individuals at UHR, regardless of transition to psychosis. Kim et al. showed that the amplitude of $\mathrm{MMN}$ in response to duration deviant stimuli (dMMN) predicted later remission, improvement in attenuated positive symptoms, and functional recovery (20). However, longitudinal studies using other deviant stimuli, such as intensity and frequency, have not been reported. Because the association between MMN to frequency deviant stimuli (fMMN) and global functioning differs from that between $\mathrm{dMMN}$ and global functioning (21), both fMMN and dMMN should be investigated. Further, neurocognitive function is also important since it mediates the association between MMN and functional outcomes in chronic schizophrenia (22). Therefore, the aim of this study was to investigate whether both $\mathrm{dMMN}$ and $\mathrm{fMMN}$ can predict future remission and neurocognitive function in individuals at UHR for psychosis.

\section{MATERIALS AND METHODS}

\section{Participants}

This study was performed as part of our multimodal research project to investigate biomarkers for psychosis [IN-STEP: the Integrative Neuroimaging Studies in Schizophrenia Targeting for Early Intervention and Prevention (23)]. At study enrollment, 39 out of 53 participants recruited to IN-STEP as individuals at UHR underwent EEG recording; among them, 24 underwent 
follow up. The current study enrolled 24 individuals at UHR and 18 healthy controls (HC). Among these 42 participants, 41 had participated in our previous ERP studies (21, 24-26).

Individuals at UHR were recruited from the outpatient and inpatient units at the University of Tokyo Hospital while HC participants were recruited through advertisements at several universities in Tokyo. The inclusion criteria of individuals at UHR were: aged 12-30 years; a history of antipsychotic $\leq 16$ cumulative weeks at the enrollment time in the IN-STEP project; and confirmed as being at UHR using the Structured Interview for Prodromal Symptoms (SIPS) $(27,28)$. The inclusion criteria of HCs were: aged 12-40 years; no history of psychiatric disease [confirmed using the Japanese version of the Mini International Neuropsychiatric Interview (29)]; and no family history of firstdegree relatives diagnosed with an axis I disorder based on the criteria of the Diagnostic and Statistical Manual of Mental Disorders, Fourth Edition (DSM-IV) (30). The exclusion criteria for all participants were any neurological illness, traumatic brain injury with cognitive consequences or loss of consciousness for $>5$ minutes, a history of electroconvulsive therapy, a low premorbid intelligence quotient [IQ; < 70 as estimated using the Japanese version of the National Adult Reading Test $(31,32)]$, previous alcohol or substance abuse or addiction, and hearing impairment revealed by audiometer testing in both ears at a $30 \mathrm{~dB}$ sound pressure level and a tone frequency of $1,000 \mathrm{~Hz}$ and $40 \mathrm{~dB}$ at 4,000 $\mathrm{Hz}$. Inclusion and exclusion criteria have been described in greater detail by Koike et al. (23). Written informed consent in accordance with the Declaration of Helsinki was obtained from each participant before enrolment in the study. For participants aged $<20$ years, written informed assent and consent were obtained from the participant and his/her parents, respectively. This study was approved by the Research Ethics Committee of the Faculty of Medicine of the University of Tokyo (approval no. 629 and 2226).

At baseline, all of the participants underwent electroencephalogram (EEG) recording, and their global and neurocognitive functions were assessed by the Global Assessment of Functioning (GAF) (30) and the Brief Assessment of Cognition in Schizophrenia (BACS) $(33,34)$ scales, respectively. For individuals at UHR, psychotic symptoms were also measured by the Scale of Prodromal Symptoms (SOPS) positive subscale included in the SIPS assessment at baseline (Time 1). Further, individuals at UHR underwent assessment at $>180$ days after baseline (Time 2) to determine prognosis, including the transition to psychosis, using the GAF and BACS scales, as well as the SIPS/SOPS (individuals who had transitioned to psychosis were not assessed by SOPS at Time 2). Remission from UHR status was defined by a score of $\geq 61$ on the GAF and $\leq 2$ on all SOPS positive subscales at the last follow-up time-point without transitioning to psychosis, according to previous studies $(20,35)$. For individuals at UHR, the prescribed antipsychotic drug doses were converted to chlorpromazine equivalent doses (36). Moreover, we examined the duration of untreated prodromal psychosis, which is the period between the appearance of the first prodromal symptom and the first hospital visit.

\section{Stimuli and Procedure}

Two auditory oddball paradigms using duration and frequency deviant stimuli were employed. For dMMN, 2,000 stimuli consisting of $90 \%$ standard tones $(1,000 \mathrm{~Hz}, 50 \mathrm{~ms})$ and $10 \%$ deviant tones $(1,000 \mathrm{~Hz}, 100 \mathrm{~ms})$ were used. For fMMN, 2,000 stimuli consisting of $90 \%$ standard tones $(1,000 \mathrm{~Hz}, 50 \mathrm{~ms})$ and $10 \%$ deviant tones $(1,200 \mathrm{~Hz}, 50 \mathrm{~ms})$ were used. The order of the two paradigms was counterbalanced across participants. All stimuli were presented binaurally through earphones while participants sat watching a silent cartoon. The auditory parameters were delivered at an $80-\mathrm{dB}$ sound pressure level, 1 $\mathrm{ms}$ rise/fall time, and $500 \mathrm{~ms}$ stimulus-onset asynchrony.

\section{EEG Recording and Analyses}

EEG data were recorded using a 64-channel Geodesic EEG System (Electrical Geodesics Inc., Eugene, OR). The electrodes were referenced to the vertex with the impedances being maintained $<50 \mathrm{k} \Omega$. The sampling rate was set at $500 \mathrm{~Hz}$ with the analog filter bandpass set at 0.1 to $100 \mathrm{~Hz}$.

The data were analyzed with EEGLAB, which is an opensource toolbox for EEG analysis (37). EEG signals at each electrode were re-referenced using an average reference and digitally filtered at $0.1-20 \mathrm{~Hz}$. Epochs were extracted from -100 to $500 \mathrm{~ms}$ relative to the stimulus onset; further, the baseline was corrected by subtracting the mean amplitude from -100 to $0 \mathrm{~ms}$. Eyeblink artifacts were corrected through independent component analysis. Epochs exceeding $\pm 100 \mu \mathrm{V}$ at each electrode were excluded. ERP waveforms for both standard and deviant stimuli were obtained through across-trial averaging. The MMN waveform was obtained as the difference in the average waveforms between the standard and deviant stimuli. We computed the $\mathrm{dMMN}$ and $\mathrm{AMMN}$ amplitudes as the mean amplitudes from 135 to $205 \mathrm{~ms}$ and from 100 to $200 \mathrm{~ms}$ post-stimulus, respectively, as we previously reported $(21,24-$ 26).To analyze the MMN amplitude, we selected seven electrodes around the frontocentral electrode $\mathrm{FCz}$ (Geodesic Sensor Net (GSN) numbers: 3, 4, 5, 8, 9, 55, and 58) where the largest MMN amplitude was obtained (the selected electrodes were shown as white circles in the topographic maps in Figures 1 and 2). The average amplitude of these seven electrodes was used as the MMN amplitude for each participant.

\section{Statistical Analysis}

IBM $^{\circledR}$ Statistical Package for the Social Sciences (SPSS ${ }^{\circledR}$ ) Statistics version 25 (IBM Corp., New York, USA) was used for all statistical analyses. Chi-squared tests and t-tests were used for comparing categorical and continuous variables of demographic characteristics between the UHR and HC groups at Time 1. When the variances were not equal, Welch's t-test was used instead of an independent $t$ test. For individuals at UHR, clinical characteristics between Time 1 and Time 2 were compared using paired t-tests to assess longitudinal changes. We compared demographic characteristics, clinical characteristics, and MMN amplitude at Time 1 between the 24 and 15 individuals at UHR who were followed and not followed up at Time 2, respectively.

Baseline demographic and clinical characteristics, including $\mathrm{dMMN}$ and $\mathrm{fMMN}$ amplitudes, were compared between the UHR-R and UHR-NR subgroups using Fisher's exact tests and Mann-Whitney U-tests for categorical and continuous variables, respectively. To evaluate whether baseline MMN amplitudes could predict functional, symptomatic, and neurocognitive 
A

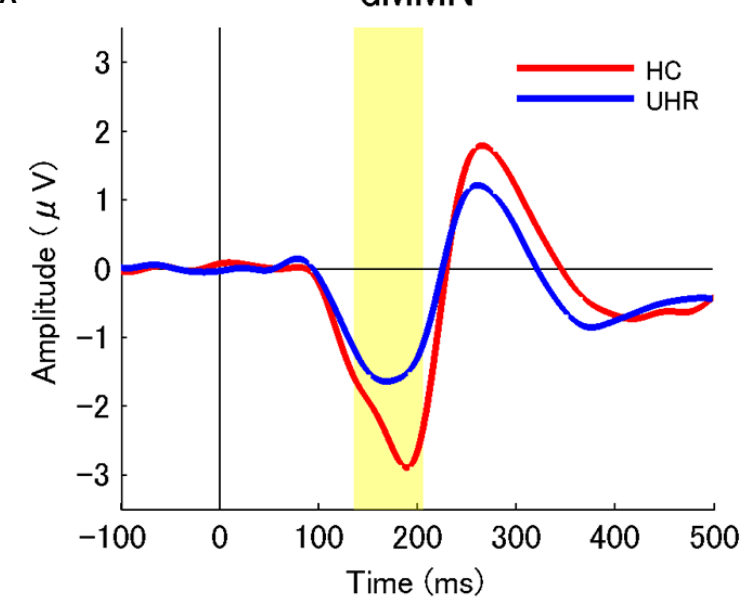

B

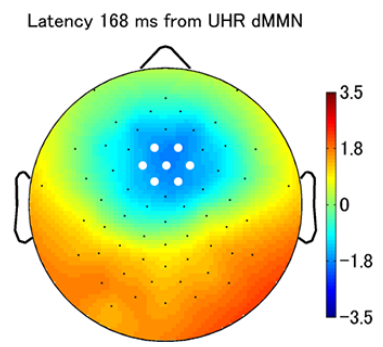

dMMN

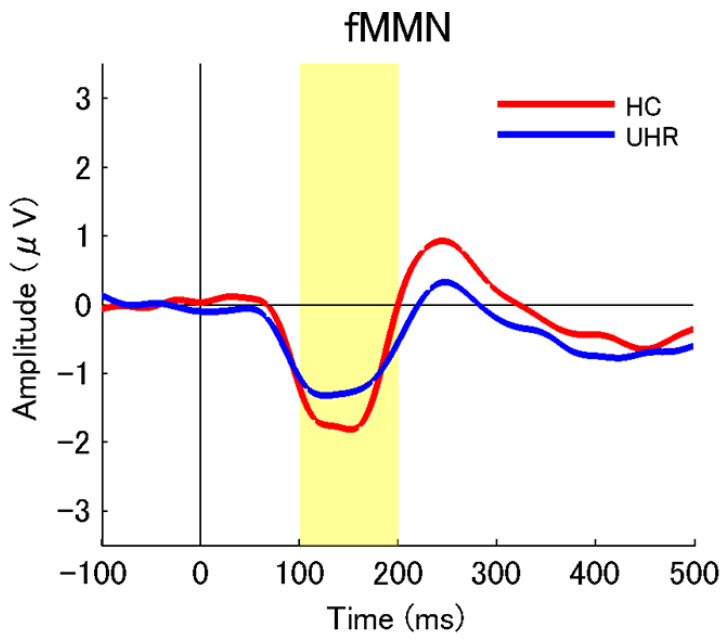

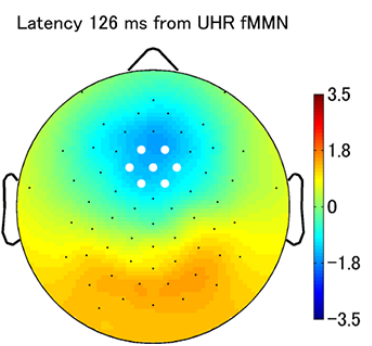

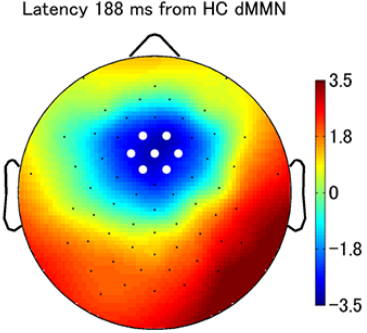

Latency $152 \mathrm{~ms}$ from HC fMMN

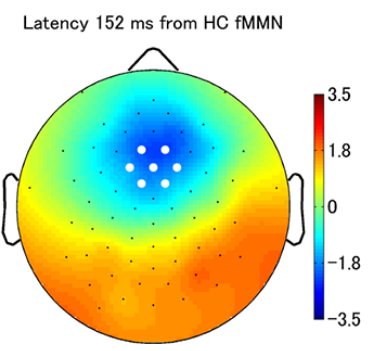

FIGURE 1 | (A) The average waveforms for duration mismatch negativity (dMMN; left) and frequency mismatch negativity (fMMN; right) at the seven electrodes around the frontocentral electrode ( $F C z$ ) in individuals at ultra-high risk for psychosis (UHR; blue line) and healthy control (HC) participants (red line). Between-group differences in the $\mathrm{dMMN}$ amplitudes were significant at the.01 level. (B) Two-dimensional topographic maps at the latency of peak amplitudes of dMMN (left) and fMMN (right) in both groups. The latency of peak amplitudes were $168 \mathrm{~ms}$ (dMMN, UHR), $188 \mathrm{~ms}(\mathrm{dMMN}, \mathrm{HC}), 126 \mathrm{~ms}$ (fMMN, UHR), and $152 \mathrm{~ms}$ (fMMN, HC).

White circles represent the seven electrodes around the FCz.

prognosis, we performed a multiple regression analysis using the stepwise selection method. Here, we used the following independent variables: both $\mathrm{dMMN}$ and $\mathrm{fMMN}$ amplitudes at baseline; demographic characteristics, including sex, age at baseline, and premorbid IQ; GAF score and total SOPS positive subscale score at baseline; follow-up period; and antipsychotics use. We adopted the GAF score; total SOPS positive subscale scores; and each BACS subscore, including the composite score, at Time 2 as the dependent variables. Statistical significance was set at .05 .

\section{RESULTS}

\section{Demographic and Clinical Characteristics}

The demographic and clinical characteristics at baseline (Time 1) are shown in Table 1. Individuals at UHR were categorized based on whether they met the criteria for BIPS, APS, and GRDS. The number individuals at UHR who met the aforementioned criteria were: 2 (8\%) for BIPS only; 16 (67\%) for APS only; 1 (4\%) for GRDS only; and $5(21 \%)$ for APS + GRDS. None of the individuals at UHR met the remission criteria at baseline. GAF scores were significantly different between the UHR and HC groups $\left(t_{28.9}=-20.46, p<0.001\right)$. Moreover, the dMMN amplitude at baseline in the UHR group was significantly attenuated compared to that in the HC group $\left(\mathrm{t}_{40}=3.13, p=\right.$ 0.003 ) while there was no significant between-group difference in the fMMN amplitudes. Figure 1 shows the grand-average waveforms and two-dimensional topographic maps of both $\mathrm{dMMN}$ and $\mathrm{fMMN}$ at baseline in both groups. There was no significant difference in the demographic or clinical characteristics at baseline between individuals with and without follow up at Time 2. The Supplementary Table shows the comparison between individuals at UHR with and without follow-up.

\section{Longitudinal Changes in Clinical Characteristics}

Table 2 shows the longitudinal changes in clinical characteristics in individuals at UHR. The average follow-up time was 604 days (standard deviation: 297 days; range: 185-1,133 days). Further, 3 out of the 24 individuals at UHR developed psychosis during the follow-up period, all of whom were diagnosed with schizophrenia based on the DSM-IV criteria. GAF scores improved over time, 
A

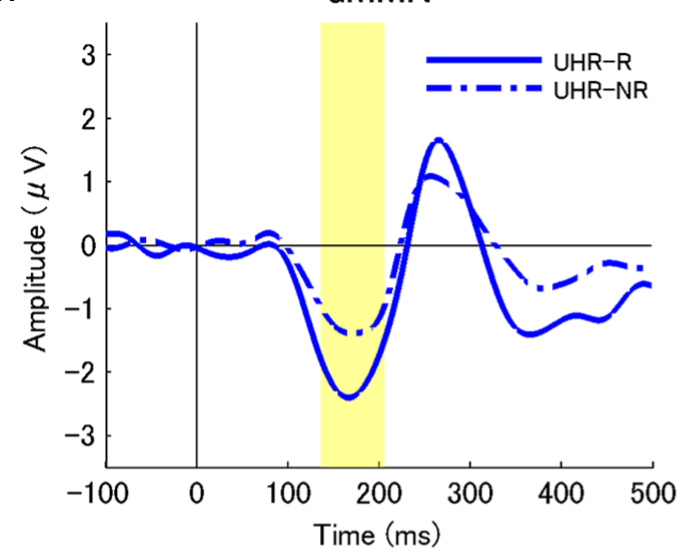

B

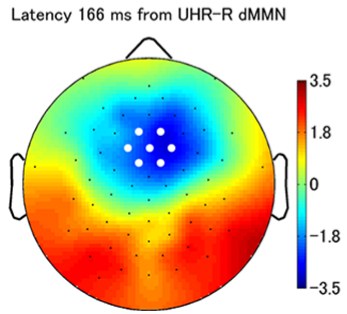

dMMN

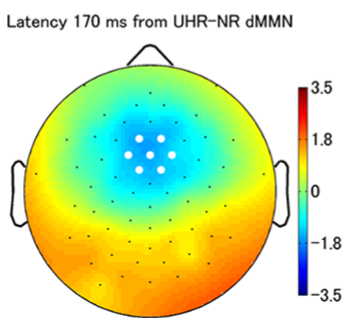

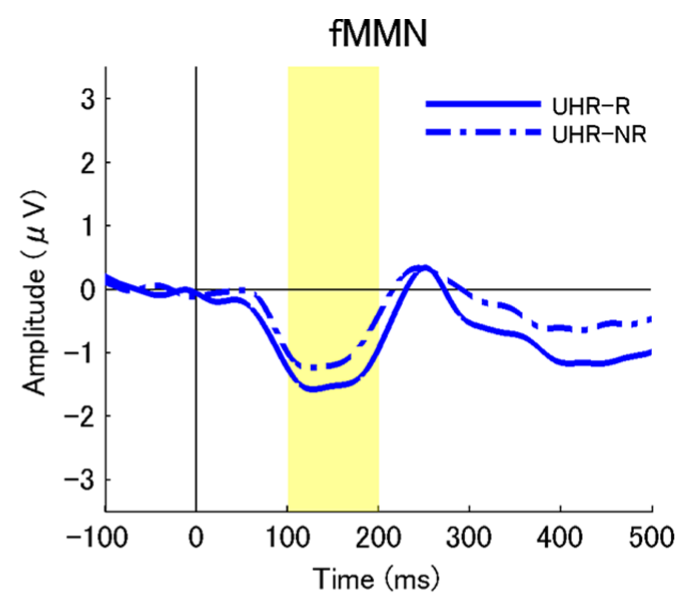
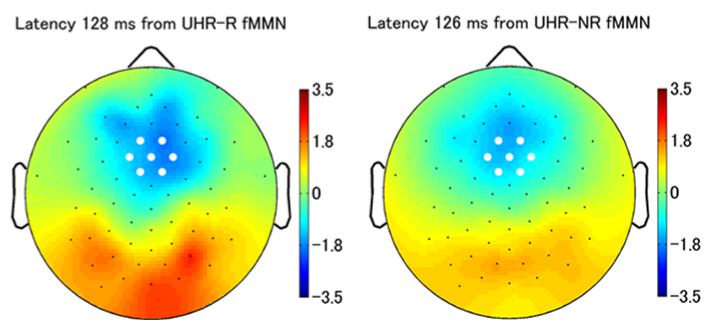

FIGURE 2 | (A) The average waveforms for duration mismatch negativity (dMMN; left) and frequency mismatch negativity (fMMN; right) at the seven electrodes around the frontocentral electrode (FCz) at baseline (Time 1) in individuals at ultra-high risk for psychosis (UHR) who achieved remission (remitters) (UHR-R; remitted at Time 2; $N$ = 6; blue solid line) and those who did not (non-remitters) (UHR-NR; not remitted at Time 2; $N$ = 18; blue chain line). There were significant betweensubgroup differences in the dMMN amplitudes at the.05 level. (B) Two-dimensional topographic maps of the latency of peak amplitudes of dMMN (left) and fMMN (right) in both subgroups. The latency of the peak amplitudes were $166 \mathrm{~ms}$ (dMMN, UHR-R), $170 \mathrm{~ms}$ (dMMN, UHR-NR), $128 \mathrm{~ms}$ (fMMN, UHR-R), and $126 \mathrm{~ms}$ (fMMN, UHR-NR), respectively. White circles represent the seven electrodes around the FCz.

TABLE 1 | Demographic and clinical characteristics of the participants at baseline (Time 1).

\begin{tabular}{|c|c|c|c|c|}
\hline \multirow{2}{*}{$\begin{array}{l}\text { Sex (Male/Female) } \\
\text { Age }(\text { years) })^{\mathrm{b}, \mathrm{c}}\end{array}$} & $\begin{array}{c}\text { UHR ( } \mathbf{N}=\mathbf{2 4}) \\
12 / 12\end{array}$ & $\begin{array}{c}\mathbf{H C}(\mathbf{N}=\mathbf{1 8}) \\
10 / 8\end{array}$ & \multicolumn{2}{|c|}{ Statistics } \\
\hline & $\begin{array}{c}20.4(3.7) \\
14-28\end{array}$ & $\begin{array}{c}21.9(3.6) \\
16-30\end{array}$ & $\mathrm{t}_{40}=-1.29$ & $p=0.204$ \\
\hline Education $\left(\right.$ years) ${ }^{\mathrm{b}}$ & $12.7(2.6)$ & $13.8(2.5)$ & $t_{40}=-1.35$ & $p=0.186$ \\
\hline Premorbid $\mathrm{IQ}^{\mathrm{b}}$ & $105.9(8.8)$ & $107.1(9.1)$ & $t_{40}=-0.43$ & $p=0.667$ \\
\hline $\mathrm{GAF}^{\mathrm{d}}$ & $47.1(9.4)$ & $88.8(3.0)$ & $t_{28.9}=-20.46$ & $p<0.001^{\star \star \star}$ \\
\hline \multicolumn{5}{|l|}{ BACS (z score) } \\
\hline Working memory & $-0.33(1.20)$ & $0.27(0.86)$ & $\mathrm{t}_{40}=-1.79$ & $p=0.081$ \\
\hline Motor speed ${ }^{\mathrm{b}, \mathrm{e}}$ & $-0.77(1.56)$ & $0.36(1.23)$ & $t_{39}=-0.91$ & $p=0.367$ \\
\hline Verbal fluency ${ }^{b}$ & $0.00(1.48)$ & $0.20(1.45)$ & $t_{40}=-0.44$ & $p=0.661$ \\
\hline Attention ${ }^{\mathrm{b}}$ & $-0.08(1.18)$ & $0.35(0.67)$ & $t_{40}=-1.40$ & $p=0.169$ \\
\hline Executive function ${ }^{\mathrm{b}}$ & $-0.20(1.26)$ & $0.10(0.63)$ & $t_{40}=-0.91$ & $p=0.366$ \\
\hline $\mathrm{dMMN}$ amplitude $(\mu \mathrm{V})^{\mathrm{b}}$ & $-1.49(0.85)$ & $-2.36(0.94)$ & $t_{40}=3.13$ & $p=0.003^{\star \star}$ \\
\hline
\end{tabular}

All values except for sex are shown as means (standard deviation). ${ }^{* *}$ The mean difference is significant at the .01 level; ***The mean difference is significant at the .001 level. UHR, ultra-high risk for psychosis; HC, healthy control; IQ, intelligence quotient; GAF, Global Assessment of Functioning; BACS, Brief Assessment of Cognition in Schizophrenia; dMMN, duration mismatch negativity; fMMN, frequency mismatch negativity.

${ }^{a}$ Chi-square tests used for statistical comparisons.

bindependent $t$-tests used for statistical comparisons.

${ }^{c}$ The range is described in the lower row.

${ }^{d}$ Welch's t-test used for statistical comparisons.

${ }^{e}$ One HC participant had a missing BACS subscore related to motor speed. 
even for those individuals who transitioned to psychosis (mean value: 47.1 to $55.5, p=0.004$ ). Further, the SOPS positive subscale scores improved among non-converters (mean value of total SOPS positive subscales: 9.4 to $5.2, p<0.001$ ). Six individuals met the remission criteria at Time 2 . There was an improvement in the verbal memory (mean value: -0.33 to $0.25, p=0.002$ ); however, there were no improvements in the other BACS subscores, including the composite score. There were no significant differences in the chlorpromazine-equivalent doses of antipsychotic drugs between Time 1 and Time 2 .

\section{Relationship Between Baseline MMN Amplitudes and Prognosis}

The dMMN amplitude at baseline were significantly larger in the remitter group (UHR-R; $\mathrm{n}=6$ ) than in the non-remitter group (UHR-NR; $\mathrm{n}=18)(p=0.039)$. However, there were no significant differences in the fMMN amplitude between the UHR-R and UHR-NR subgroups $(p=0.096)$. Figure 2 shows the grandaverage waveforms and two-dimensional topographic maps for each group. Table 3 shows the between-subgroup differences in the baseline demographic and clinical characteristics, as well as MMN amplitudes. There was no between-subgroup difference in the GAF, SOPS positive subscale, or BACS scores, as well as the chlorpromazine-equivalent doses of antipsychotic drugs. The UHR-R group exhibited a longer follow-up period, had a younger mean age, and fewer years of education at baseline compared to the UHR-NR subgroup.

Multiple regression analysis revealed that the total SOPS positive subscales and $\mathrm{fMMN}$ amplitude at baseline predicted the BACS attention subscores at follow-up $\left(\mathrm{F}_{2,21}=4.98, \mathrm{R}^{2}=\right.$ $0.32, p=0.017$; SOPS positive subscales, beta $=0.13$ [95\% CI, 0.01 to 0.25 , standardized beta $=0.42, \mathrm{t}=2.33, p=0.030$; $\mathrm{fMMN}$, beta $=-0.85$ [ $95 \% \mathrm{CI},-1.66$ to -0.04$]$, standardized beta $=-0.39$, $\mathrm{t}=-2.18, p=0.041)$. Figure 3 shows the association between fMMN at baseline and BACS attention subscore at the follow-up point, which was adjusted for total SOPS subscales at baseline.

\section{DISCUSSION}

This study assessed whether baseline dMMN and fMMN amplitudes could predict the prognosis of individuals at UHR, regardless of whether they transitioned to psychosis. There were significantly larger dMMN amplitudes at baseline in individuals at UHR who achieved remission compared with those who did not. Further, multiple regression analysis revealed that fMMN amplitude at baseline predicted the BACS attention score at the follow-up point.

\section{Functioning and Neurocognitive Function in UHR}

Compared with the HC group, the UHR group showed significantly reduced the GAF score; however, there was no between-group difference in the BACS score. There have been numerous reports of impaired functioning in individuals at $\operatorname{UHR}(8,38)$. Moreover, a meta-analysis by Fusar-Poli et al. reported reduced functioning in the UHR group by around three in the effect size compared to HC participants (39). Further, neurocognitive function is reduced in various domains; however, the effect size is small with a maximum of around 0.5 (40). We found that neurocognitive functions were not significantly reduced in UHR. The effect sizes of neurocognitive impairments in UHR range from 0.14 to 0.79 (Cohen's $d$ ). Therefore, impaired functioning in individuals at UHR could be attributed to clinical symptoms rather than neurocognitive impairments. Specifically, improvements in positive and negative symptoms contributed to improved social and role functioning in UHR, respectively (41). Further, depression and anxiety, which are often comorbid with UHR (8), may influence functioning. However, it was previously reported that neurocognitive function could predict future functioning in $\operatorname{UHR}(38,42,43)$. Since usual treatments, including pharmacotherapy and psychotherapy, can improve clinical symptoms but not neurocognitive function, neurocognitive impairments could limit functioning improvement in individuals at UHR.

TABLE 2 | Longitudinal changes in clinical characteristics in individuals at UHR.

\begin{tabular}{|c|c|c|c|c|}
\hline $\mathrm{GAF}^{\mathrm{a}}$ & $\begin{array}{c}\text { Time 1 } \\
47.1(9.4)\end{array}$ & $\begin{array}{c}\text { Time } 2 \\
55.5(12.4)\end{array}$ & \multicolumn{2}{|c|}{ Statistics } \\
\hline Total SOPS positive subscales ${ }^{a, b}$ & $9.4(3.6)$ & $5.2(2.9)$ & $t_{20}=4.68$ & $p<0.001^{\star \star \star}$ \\
\hline Composite $^{\mathrm{a}}$ & $-0.28(0.91)$ & $-0.09(0.71)$ & $\mathrm{t}_{23}=-1.63$ & $p=0.117$ \\
\hline Verbal memory ${ }^{a}$ & $-0.33(1.20)$ & $0.25(0.95)$ & $\mathrm{t}_{23}=-3.54$ & $\mathrm{p}=0.002^{\star \star}$ \\
\hline Working memory ${ }^{\mathrm{a}}$ & $-0.33(1.20)$ & $-0.24(1.10)$ & $\mathrm{t}_{23}=-0.45$ & $p=0.655$ \\
\hline Motor speed ${ }^{\mathrm{a}}$ & $-0.77(1.56)$ & $-0.60(1.18)$ & $\mathrm{t}_{23}=-0.62$ & $p=0.543$ \\
\hline Executive function ${ }^{a}$ & $-0.20(1.26)$ & $0.29(0.99)$ & $\mathrm{t}_{23}=-1.63$ & $p=0.116$ \\
\hline Antipsychotics (mg/day) ${ }^{a, c}$ & $113.6(146.7)$ & $204.6(359.1)$ & $t_{23}=-1.32$ & $p=0.199$ \\
\hline
\end{tabular}

All values are shown as means (standard deviation). ${ }^{* *}$ The mean difference is significant at the .01 level; ${ }^{* * *}$ The mean difference is significant at the .001 level.

UHR, ultra-high risk for psychosis; GAF, Global Assessment of Functioning; SOPS, Scale of Prodromal Symptoms; BACS, Brief Assessment of Cognition in Schizophrenia; CP, Chlorpromazine.

${ }^{a}$ Paired t-test used for statistical comparison.

${ }^{b}$ Except for three individuals who had progressed to psychosis.

${ }^{c}$ Reported as chlorpromazine equivalent dose. 
TABLE 3 | Baseline demographic and clinical characteristics of the UHR-R and UHR-NR subgroups.

\begin{tabular}{|c|c|c|c|c|}
\hline & UHR-R (N = 6) & UHR-NR (N = 18) & & \\
\hline Follow-up period (days) ${ }^{\mathrm{b}}$ & $899.5(179.7)$ & $505.8(263.1)$ & $U_{6,18}=12.0$ & $p=0.005^{\star \star}$ \\
\hline Education (years) ${ }^{b}$ & $10.8(2.6)$ & $13.3(2.3)$ & $U_{6,18}=23.5$ & $p=0.040^{*}$ \\
\hline Premorbid $I^{\mathrm{b}}$ & $101.3(9.2)$ & $107.4(8.4)$ & $U_{6,18}=30.5$ & $p=0.114$ \\
\hline DUPP (days) ${ }^{\mathrm{b}, \mathrm{c}}$ & $312.3(437.9)$ & $307.4(500.3)$ & $U_{6,16}=44.0$ & $p=0.768$ \\
\hline $\mathrm{GAF}^{\mathrm{b}}$ & $50.8(6.3)$ & $45.8(10.0)$ & $U_{6,18}=32.5$ & $p=0.150$ \\
\hline Composite $^{\mathrm{b}}$ & $0.10(0.83)$ & $-0.41(0.92)$ & $U_{6,18}=33.0$ & $p=0.162$ \\
\hline Verbal memory ${ }^{\mathrm{b}}$ & $0.08(0.87)$ & $-0.46(1.28)$ & $U_{6,18}=42.0$ & $p=0.423$ \\
\hline Working memory ${ }^{\mathrm{b}}$ & $-0.51(1.26)$ & $-0.27(1.22)$ & $U_{6,18}=51.5$ & $p=0.867$ \\
\hline Motor speed ${ }^{\mathrm{b}}$ & $-0.21(1.55)$ & $-0.96(1.56)$ & $U_{6,18}=36.5$ & $p=0.243$ \\
\hline Verbal fluency ${ }^{\mathrm{b}}$ & $0.56(1.86)$ & $-0.19(1.34)$ & $U_{6,18}=43.0$ & $p=0.463$ \\
\hline Attention $^{\mathrm{b}}$ & $0.40(1.04)$ & $-0.24(1.21)$ & $U_{6,18}=35.0$ & $p=0.205$ \\
\hline
\end{tabular}

All values except for sex are shown as means (standard deviation). *The mean difference is significant at the .05 level; **The mean difference is significant at the .01 level.

UHR, ultra-high risk for psychosis; UHR-R, UHR remitters; UHR-NR, UHR non-remitters; IQ, intelligence quotient; DUPP, duration of untreated prodromal psychosis; GAF, Global Assessment of Functioning; SOPS, Scale of Prodromal Symptoms; BACS, Brief Assessment of Cognition in Schizophrenia; CP, Chlorpromazine; dMMN, duration mismatch negativity; fMMN, frequency mismatch negativity.

aFisher's exact test used for statistical comparison.

${ }^{b}$ Mann-Whitney U-test used for statistical comparison.

${ }^{c}$ Two participants in the UHR-NR participant have missing DUPP data.

${ }^{d}$ Reported as chlorpromazine equivalent dose.

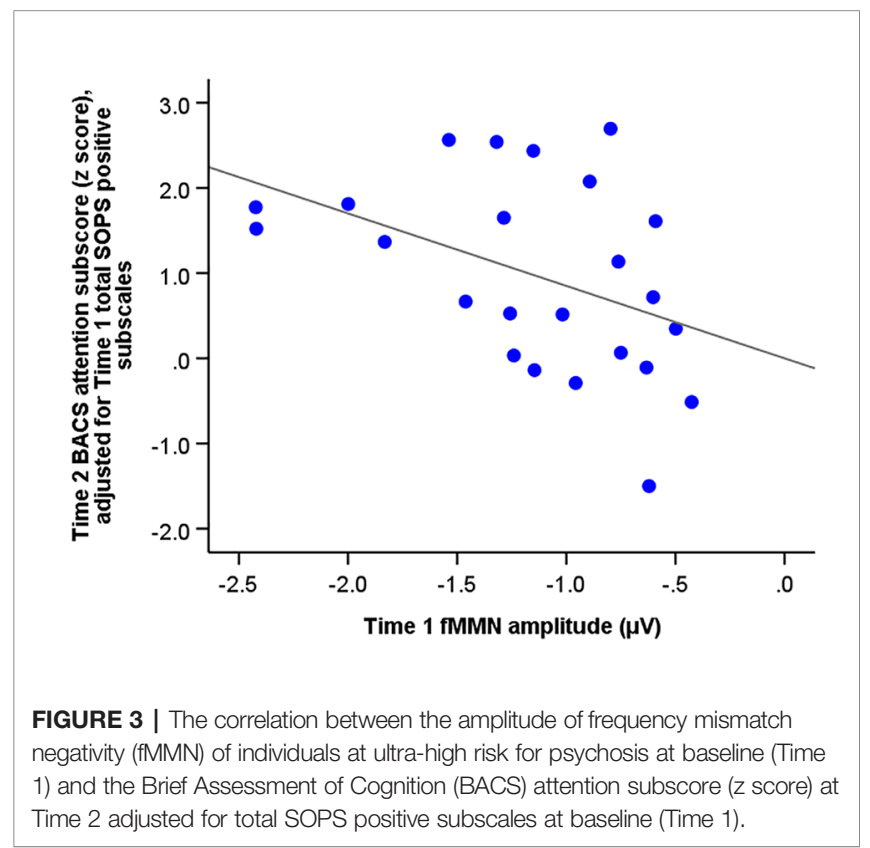

\section{Future Remission Prediction by Duration MMN}

Compared with the UHR-NR group, the UHR-R group showed a large dMMN, but not fMMN. This indicates that dMMN may predict future remission in UHR, which is consistent with previous reports that $\mathrm{dMMN}$ can predict future remission in individuals at UHR (20). In addition, these findings indicate that prediction of future remission in individuals at UHR by MMN is dependent on deviant type.

Previous cross-sectional studies have reported an association of dMMN with functioning in schizophrenia $(21,44)$ and UHR (21, $45)$. Several studies $(21,45,46)$, including this study, have reported reduced dMMN amplitude compared with HC. Moreover, neural circuits underlying dMMN could be impaired in UHR, which may not only affect current functioning but also future remission. For example, Kim et al. reported reduced dMMN current source density in the right frontal cortex and functional disconnection between the temporal and frontal cortices in UHR (46). Given the previous reports of MMN generators in multiple cortical sources (47) and connectivity among the cortical sources (48), these neural circuits could attribute to reduced $\mathrm{dMMN}$ and prediction of future remission in UHR. However, there is a need for further studies to clarify neural circuits underlying reduced $\mathrm{dMMN}$ and future remission prediction in UHR.

\section{Prediction of Future Neurocognitive Function by Frequency MMN}

We found that the fMMN amplitude along with the baseline positive symptoms predicted future neurocognitive function of attention. Although individuals at UHR presented intact fMMN as a group, inter-individual differences in fMMN could affect future neurocognitive function. To our knowledge, this is the first study to report that $\mathrm{PMMN}$ can predict the UHR prognosis.

In schizophrenia, impaired early auditory information processing, as indicated by reduced MMN, could affect neurocognitive deficits, which results in subsequent poor functional outcomes (22). However, previous cross-sectional 
studies have reported inconsistent findings regarding the correlations between $\mathrm{MMN}$ and neurocognitive function in UHR. Higuchi et al. reported a correlation between dMMN and verbal fluency in UHR (49). Moreover, Carrión et al. reported a correlation between fMMN and processing speed across individuals at UHR and HCs (45). A previous crosssectional study of our group reported no significant correlation of either dMMN or $\mathrm{AMMN}$ with neurocognitive function in UHR (21). In this study, there was no correlation of MMN amplitudes with each BACS subscore at baseline. These current findings indicate that $\mathrm{fMMN}$ may be associated with future, but not current, neurocognitive function.

Contrastingly, the attention neurocognition domain, which we found to be associated with $\mathrm{PMMN}$, has been found to predict functioning. Sawada et al. reported that BACS attention subscores could predict future modified GAF scores in individuals at UHR who do not develop psychosis (43). The attention subdomain of the BACS is measured using the symbol coding task. Further, the processing speed, which is indicated by similar digit symbol-coding subtests and Trail Making Test, could predict social functioning (38, 42). Therefore, fMMN may affect functional prognosis through neurocognitive function. However, we could not perform, for example, structural equation modeling to investigate the $\mathrm{AMMN}$ effect on the future functional outcome through neurocognitive function given the small sample size. There is a need for further studies to clarify this.

\section{Limitations}

This study had several limitations. First, we adopted a naturalistic design and did not control for the duration of the follow-up period or any medications used by the participants. The inter-individual difference in the follow-up period and medication use could have biased and affected the results. The impact of various psychotropic drugs on MMN remains unclear (50); however, antipsychotics and benzodiazepines have been shown not to affect $\operatorname{MMN}(51,52)$. Second, some of the individuals at UHR had relatively short follow-up periods. Although the mean follow-up period was around 2 years, the follow-up period was $<1$ year in 6 out of the 24 individuals at UHR. Following up on these 6 individuals at UHR for longer periods could have changed their prognosis. Finally, there were unavailable MMN data upon follow-up. Only 10 out of the 24 individuals at UHR had MMN data at the follow-up point; moreover, the sample size was insufficient for analyses. The availability of MMN data at both baseline and follow-up points could contribute to a more comprehensive understanding of the longitudinal association between MMN and prognosis.

\section{CONCLUSION}

In conclusion, we observed an association between future remission and $\mathrm{dMMN}$ in individuals at UHR. Moreover, we found that $\mathrm{PMMN}$ predicted neurocognitive function in UHR. These findings suggest that both $\mathrm{dMMN}$ and $\mathrm{fMMN}$ could be candidate biomarkers for predicting the prognosis of individuals at UHR.

\section{DATA AVAILABILITY STATEMENT}

The raw data supporting the conclusions of this article may be available upon request only if the ethical committee of Faculty of Medicine, the University of Tokyo approves it.

\section{ETHICS STATEMENT}

The studies involving human participants were reviewed and approved by the Research Ethics Committee of the Faculty of Medicine, The University of Tokyo. Written informed consent to participate in this study was provided by the participants or their parents.

\section{AUTHOR CONTRIBUTIONS}

KKi, DK, MT, and TN collected the data. MF, KKi, DK, MT, TN, and KU analyzed the data. MF, KKi, DK, MT, and KU interpreted the results. SKo, MS, TA, and KKa designed the study. KKa supervised all aspects of data collection, analysis, and interpretation. $\mathrm{MF}, \mathrm{KKi}$, and $\mathrm{KKa}$ wrote the first draft of the manuscript. All the authors commented on the manuscript. All authors contributed to the article and approved the submitted version.

\section{FUNDING}

This study was supported in part by the Japan Agency for Medical Research and development, AMED (grant JP20dm0207069), Japan Society for the Promotion of Science, JSPS KAKENHI (grants JP16H06395, JP16H06399, and JP16K21720), Takeda Science Foundation, UTokyo Center for Integrative Science of Human Behavior (CiSHuB), and the International Research Center for Neurointelligence (WPIIRCN) at The University of Tokyo Institutes for Advanced Study (UTIAS).

\section{ACKNOWLEDGMENTS}

We appreciate the members of the Integrative Neuroimaging Studies for Schizophrenia Targeting Early Intervention and Prevention (IN-STEP) research team at the University of Tokyo Hospital for providing advice and supporting this project. We also appreciate all the study participants.

\section{SUPPLEMENTARY MATERIAL}

The Supplementary Material for this article can be found online at: https://www.frontiersin.org/articles/10.3389/fpsyt.2020.00770/ full\#supplementary-material 


\section{REFERENCES}

1. Penttilä M, Jääskeläinen E, Hirvonen N, Isohanni M, Miettunen J. Duration of untreated psychosis as predictor of long-term outcome in schizophrenia : systematic review and meta-analysis. Br J Psychiatry (2014) 205:88-94. doi: 10.1192/bjp.bp.113.127753

2. Yung AR, Phillips LJ, Yuen HP, Francey SM, McFarlane CA, Hallgren M, et al. Psychosis prediction: 12-month follow up of a high-risk ("prodromal") group. Schizophr Res (2003) 60:21-32. doi: 10.1016/S0920-9964(02)00167-6

3. Miller TJ, McGlashan TH, Rosen JL, Cadenhead K, Ventura J, McFarlane W, et al. Prodromal assessment with the structured interview for prodromal syndromes and the scale of prodromal symptoms: Predictive validity, interrater reliability, and training to reliability. Schizophr Bull (2003) 29:703-15. doi: 10.1093/oxfordjournals.schbul.a007040

4. Fusar-Poli P, Bonoldi I, Yung AR, Borgwardt S, Kempton MJ, Valmaggia L, et al. Predicting psychosis. Arch Gen Psychiatry (2012) 69:220-9. doi: 10.1001/ archgenpsychiatry.2011.1472

5. Simon AE, Borgwardt S, Riecher-Rössler A, Velthorst E, de Haan L, FusarPoli P. Moving beyond transition outcomes: Meta-analysis of remission rates in individuals at high clinical risk for psychosis. Psychiatry Res (2013) 209:266-72. doi: 10.1016/j.psychres.2013.03.004

6. de Wit S, Schothorst PF, Oranje B, Ziermans TB, Durston S, Kahn RS. Adolescents at ultra-high risk for psychosis: Long-term outcome of individuals who recover from their at-risk state. Eur Neuropsychopharmacol (2014) 24:865-73. doi: 10.1016/j.euroneuro.2014.02.008

7. de Wit S, Wierenga LM, Oranje B, Ziermans TB, Schothorst PF, van Engeland H, et al. Brain development in adolescents at ultra-high risk for psychosis: Longitudinal changes related to resilience. NeuroImage Clin (2016) 12:542-9. doi: 10.1016/j.nicl.2016.08.013

8. Addington J, Cornblatt BA, Cadenhead KS, Cannon TD, McGlashan TH, Perkins DO, et al. At clinical high risk for psychosis: Outcome for nonconverters. Am J Psychiatry (2011) 168:800-5. doi: 10.1176/appiajp. 2011.10081191

9. Bodatsch M, Brockhaus-Dumke A, Klosterkötter J, Ruhrmann S. Forecasting psychosis by event-related potentials - Systematic review and specific metaanalysis. Biol Psychiatry (2015) 77:951-8. doi: 10.1016/j.biopsych.2014.09.025

10. Light GA, Swerdlow NR. Future clinical uses of neurophysiological biomarkers to predict and monitor treatment response for schizophrenia. Ann N Y Acad Sci (2015) 1344:105-19. doi: 10.1111/nyas.12730

11. Erickson MA, Ruffle A, Gold JM. A meta-analysis of mismatch negativity in schizophrenia: From clinical risk to disease specificity and progression. Biol Psychiatry (2016) 79:980-7. doi: 10.1016/j.biopsych.2015.08.025

12. Javitt DC, Steinschneider M, Schroeder CE, Arezzo JC. Role of cortical Nmethyl-D-aspartate receptors in auditory sensory memory and mismatch negativity generation: Implications for schizophrenia. Proc Natl Acad Sci U.S.A. (1996) 93:11962-7. doi: 10.1073/pnas.93.21.11962

13. Rosburg T, Kreitschmann-Andermahr I. The effects of ketamine on the mismatch negativity (MMN) in humans - A meta-analysis. Clin Neurophysiol (2016) 127:1387-94. doi: 10.1016/j.clinph.2015.10.062

14. Miyanishi T, Sumiyoshi T, Higuchi Y, Seo T, Suzuki M. LORETA current source density for duration mismatch negativity and neuropsychological assessment in early schizophrenia. PloS One (2013) 8:e61152. doi: 10.1371/ journal.pone.0061152

15. Toyomaki A, Kusumi I, Matsuyama T, Kako Y, Ito K, Koyama T. Tone duration mismatch negativity deficits predict impairment of executive function in schizophrenia. Prog Neuropsychopharmacol Biol Psychiatry (2008) 32:95-9. doi: 10.1016/j.pnpbp.2007.07.020

16. Light GA, Braff DL. Mismatch negativity deficits are associated with poor functioning in schizophrenia patients. Arch Gen Psychiatry (2005) 62:127-36. doi: 10.1001/archpsyc.62.2.127

17. Rasser PE, Schall U, Todd J, Michie PT, Ward PB, Johnston P, et al. Gray matter deficits, mismatch negativity, and outcomes in schizophrenia. Schizophr Bull (2011) 37:131-40. doi: 10.1093/schbul/sbp060

18. Nagai T, Tada M, Kirihara K, Araki T, Jinde S, Kasai K. Mismatch negativity as a "translatable" brain marker toward early intervention for psychosis: A review. Front Psychiatry (2013) 4:115:115. doi: 10.3389/fpsyt.2013.00115

19. Tada M, Kirihara K, Mizutani S, Uka T, Kunii N, Koshiyama D, et al. Mismatch negativity (MMN) as a tool for translational investigations into early psychosis: A review. Int J Psychophysiol (2019) 145:5-14. doi: 10.1016/ j.ijpsycho.2019.02.009

20. Kim M, Lee TH, Yoon YB, Lee TY, Kwon JS. Predicting remission in subjects at clinical high risk for psychosis using mismatch negativity. Schizophr Bull (2018) 44:575-83. doi: 10.1093/schbul/sbx102

21. Koshiyama D, Kirihara K, Tada M, Nagai T, Fujioka M, Koike S, et al. Association between mismatch negativity and global functioning is specific to duration deviance in early stages of psychosis. Schizophr Res (2018) 195:37884. doi: 10.1016/j.schres.2017.09.045

22. Thomas ML, Green MF, Hellemann G, Sugar CA, Tarasenko M, Calkins ME, et al. Modeling deficits from early auditory information processing to psychosocial functioning in schizophrenia. JAMA Psychiatry (2017) 74:3746. doi: 10.1001/jamapsychiatry.2016.2980

23. Koike S, Takano Y, Iwashiro N, Satomura Y, Suga M, Nagai T, et al. A multimodal approach to investigate biomarkers for psychosis in a clinical setting: The integrative neuroimaging studies in schizophrenia targeting for early intervention and prevention (IN-STEP) project. Schizophr Res (2013) 143:116-24. doi: 10.1016/j.schres.2012.11.012

24. Nagai T, Tada M, Kirihara K, Yahata N, Hashimoto R, Araki T, et al. Auditory mismatch negativity and P3a in response to duration and frequency changes in the early stages of psychosis. Schizophr Res (2013) 150:547-54. doi: 10.1016/ j.schres.2013.08.005

25. Nagai T, Kirihara K, Tada M, Koshiyama D, Koike S, Suga M, et al. Reduced mismatch negativity is associated with increased plasma level of glutamate in first-episode psychosis. Sci Rep (2017) 7:2258. doi: 10.1038/s41598-01702267-1

26. Koshiyama D, Kirihara K, Tada M, Nagai T, Koike S, Suga M, et al. Duration and frequency mismatch negativity shows no progressive reduction in early stages of psychosis. Schizophr Res (2017) 190:32-8. doi: 10.1016/ j.schres.2017.03.015

27. Miller TJ, McGlashan TH, Woods SW, Stein K, Driesen N, Corcoran CM, et al. Symptom assessment in schizophrenic prodromal states. Psychiatr Q (1999) 70:273-87. doi: 10.1023/a:1022034115078

28. Kobayashi H, Nozaki S, Mizuno M. Reliability of the structured interview for prodromal syndromes Japanese version (SIPS-J). JPN Bull Soc Psychiat (2007) 15:168-74.

29. Otsubo T, Tanaka K, Koda R, Shinoda J, Sano N, Tanaka S, et al. Reliability and validity of Japanese version of the Mini-International Neuropsychiatric Interview. Psychiatry Clin Neurosci (2005) 59:517-26. doi: 10.1111/j.14401819.2005.01408.x

30. American Psychiatric Association. Diagnostic and Statistical Manual of Mental Disorders. 4th ed. Washington, DC: American Psychiatric Press (1994).

31. Matsuoka K, Kim Y. Japanese adult reading test (JART). Tokyo: ShinkouIgaku publishers (2006).

32. Matsuoka K, Uno M, Kasai K, Koyama K, Kim Y. Estimation of premorbid IQ in individuals with Alzheimer's disease using Japanese ideographic script (Kanji) compound words: Japanese version of National Adult Reading Test Psychiatry Clin Neurosci (2006) 60:332-9. doi: 10.1111/j.1440-1819.2006. 01510.x

33. Keefe RSE, Goldberg TE, Harvey PD, Gold JM, Poe MP, Coughenour L. The brief assessment of cognition in schizophrenia: Reliability, sensitivity, and comparison with a standard neurocognitive battery. Schizophr Res (2004) 68:283-97. doi: 10.1016/j.schres.2003.09.011

34. Kaneda Y, Sumiyoshi T, Keefe R, Ishimoto Y, Numata S, Ohmori T. Brief assessment of cognition in schizophrenia: Validation of the Japanese version. Psychiatry Clin Neurosci (2007) 61:602-9. doi: 10.1111/j.1440-1819.2007.01725.x

35. Lee TY, Kim SN, Correll CU, Byun MS, Kim E, Jang JH, et al. Symptomatic and functional remission of subjects at clinical high risk for psychosis: A 2year naturalistic observational study. Schizophr Res (2014) 156:266-71. doi: 10.1016/j.schres.2014.04.002

36. Inada T, Inagaki A. Psychotropic dose equivalence in Japan. Psychiatry Clin Neurosci (2015) 69:440-7. doi: 10.1111/pcn.12275

37. Delorme A, Makeig S. EEGLAB: An open source toolbox for analysis of singletrial EEG dynamics including independent component analysis. J Neurosci Methods (2004) 134:9-21. doi: 10.1016/j.jneumeth.2003.10.009

38. Carrión RE, Goldberg TE, McLaughlin D, Auther AM, Correll CU, Cornblatt BA. Impact of neurocognition on social and role functioning in individuals at 
clinical high risk for psychosis. Am J Psychiatry (2011) 168:806-13. doi: 10.1176/appi.ajp.2011.10081209

39. Fusar-Poli P, Rocchetti M, Sardella A, Avila A, Brandizzi M, Caverzasi E, et al. Disorder, not just state of risk: Meta-analysis of functioning and quality of life in people at high risk of psychosis. Br J Psychiatry (2015) 207:198-206. doi: 10.1192/bjp.bp.114.157115

40. Fusar-Poli P, Deste G, Smieskova R, Barlati S, Yung AR, Howes O, et al. Cognitive functioning in prodromal psychosis. Arch Gen Psychiatry (2012) 69:562-71. doi: 10.1001/archgenpsychiatry.2011.1592

41. Niendam TA, Bearden CE, Zinberg J, Johnson JK, O’Brien M, Cannon TD. The course of neurocognition and social functioning in individuals at ultra high risk for psychosis. Schizophr Bull (2007) 33:772-81. doi: 10.1093/schbul/sbm020

42. Carrión RE, McLaughlin D, Goldberg TE, Auther AM, Olsen RH, Olvet DM, et al. Prediction of functional outcome in individuals at clinical high risk for psychosis. JAMA Psychiatry (2013) 70:1133-42. doi: 10.1001/jamapsychiatry.2013.1909

43. Sawada K, Kanehara A, Sakakibara E, Eguchi S, Tada M, Satomura Y, et al. Identifying neurocognitive markers for outcome prediction of global functioning in individuals with first-episode and ultra-high-risk for psychosis. Psychiatry Clin Neurosci (2017) 71:318-27. doi: 10.1111/pcn.12522

44. Kim M, Kim SN, Lee S, Byun MS, Shin KS, Park HY, et al. Impaired mismatch negativity is associated with current functional status rather than genetic vulnerability to schizophrenia. Psychiatry Res - Neuroimaging (2014) 222:1006. doi: 10.1016/j.pscychresns.2014.02.012

45. Carrión RE, Cornblatt BA, McLaughlin D, Chang J, Auther AM, Olsen RH, et al. Contributions of early cortical processing and reading ability to functional status in individuals at clinical high risk for psychosis. Schizophr Res (2015) 164:1-7. doi: 10.1016/j.schres.2015.01.030

46. Kim M, Cho KIK, Yoon YB, Lee TY, Kwon JS. Aberrant temporal behavior of mismatch negativity generators in schizophrenia patients and subjects at clinical high risk for psychosis. Clin Neurophysiol (2017) 128:331-9. doi: 10.1016/j.clinph.2016.11.027

47. Rissling AJ, Miyakoshi M, Sugar CA, Braff DL, Makeig S, Light GA. Cortical substrates and functional correlates of auditory deviance processing deficits in schizophrenia. NeuroImage Clin (2014) 6:424-37. doi: 10.1016/j.nicl. 2014.09.006

48. MacLean SE, Ward LM. Temporo-frontal phase synchronization supports hierarchical network for mismatch negativity. Clin Neurophysiol (2014) 125:1604-17. doi: 10.1016/j.clinph.2013.12.109

49. Higuchi Y, Sumiyoshi T, Seo T, Miyanishi T, Kawasaki Y, Suzuki M. Mismatch negativity and cognitive performance for the prediction of psychosis in subjects with at-risk mental state. PloS One (2013) 8:e54080. doi: 10.1371/journal.pone.0054080

50. Garrido MI, Kilner JM, Stephan KE, Friston KJ. The mismatch negativity: A review of underlying mechanisms. Clin Neurophysiol (2009) 120:453-63. doi: 10.1016/j.clinph.2008.11.029

51. Rissling AJ, Braff DL, Swerdlow NR, Hellemann G, Rassovsky Y, Sprock J, et al. Disentangling early sensory information processing deficits in schizophrenia. Clin Neurophysiol (2012) 123:1942-9. doi: 10.1016/ j.clinph.2012.02.079

52. Kasai K, Yamada H, Kamio S, Nakagome K, Iwanami A, Fukuda M, et al. Do high or low doses of anxiolytics and hypnotics affect mismatch negativity in schizophrenic subjects? An EEG and MEG study. Clin Neurophysiol (2002) 113:141-50. doi: 10.1016/S1388-2457(01)00710-6

Conflict of Interest: The authors declare that the research was conducted in the absence of any commercial or financial relationships that could be construed as a potential conflict of interest.

Copyright (c) 2020 Fujioka, Kirihara, Koshiyama, Tada, Nagai, Usui, Morita, Kawakami, Morita, Satomura, Koike, Suga, Araki and Kasai. This is an openaccess article distributed under the terms of the Creative Commons Attribution License (CC BY). The use, distribution or reproduction in other forums is permitted, provided the original author(s) and the copyright owner(s) are credited and that the original publication in this journal is cited, in accordance with accepted academic practice. No use, distribution or reproduction is permitted which does not comply with these terms. 